\title{
Effect of a transition section between the Blumlein line and a load on the output voltage of gigawatt intense electron-beam accelerators
}

\author{
Xin-Bing Cheng, Jin-Liang Liu, Yu Zhang, Jia-Huai Feng, and Bao-Liang Qian \\ College of Photoelectric Engineering and Science, National University of Defense Technology, Changsha, 410073, China
}

(Received 8 September 2009; published 17 November 2009)

\begin{abstract}
The flat voltage of the main pulse on the load of intense electron-beam accelerators (IEBA) is one of the important factors which affect the quality and characteristic of the output beam current of IEBA. In this paper, the effect of the transition section between the Blumlein pulse forming line (BPFL) and load on the output voltage of IEBA is analyzed in theory. Based on the telegraph equations of the BPFL, a formula which is used to calculate the output voltage of IEBA is obtained, and the factor which affects the output voltage is analyzed. It is found from the theoretical analysis that the wave impedance and transmission time of the transition section influence considerably the fluctuation of the main pulse voltage waveform at the load. In order to get a well-shaped square waveform at load and to improve the electron-beam quality of such an accelerator, there should be a proper wave impedance of the transition section, and the transmission time of the transition section should be designed as short as possible. At last, some experiments are performed on IEBA, and the experimental results show reasonable agreement with theoretical analysis.
\end{abstract}

DOI: 10.1103/PhysRevSTAB.12.110401

PACS numbers: $84.30 . \mathrm{Ng}, 84.70 .+\mathrm{p}, 87.56 . \mathrm{bd}$

\section{INTRODUCTION}

The Blumlein pulse forming line (BPFL) [1] provides flat (rectangular) voltage pulses with an amplitude equal to the charging voltage delivered into a load with a resistance, with a value matching twice the impedance of the individual pulse forming lines. The Blumlein can be constructed either in cylindrical form or in parallel plate configuration [2]. In cylindrical form [3,4], its basic structure consists of mainly three coaxial cylinders of different radii, named inner cylinder, middle cylinder, and outer cylinder, respectively. The space between the cylinders is filled with liquid dielectric medium, such as oil or water. The Blumlein can readily be designed in terms of output impedance, pulse length, and voltage by a suitable choice of the dimensions of the Blumlein cylinder conductor and the dielectric material serving as the energy storage medium.

The intense electron-beam accelerators (IEBA) based on the Blumlein line [5-7] are widely used in a great variety of applications such as in intense relativistic electron-beam generation [8], high power microwave generators (HPMG) [9], x-ray generation [10], and so on. At the same time, improving electron-beam quality is very important for the requirement of these applications. Especially in the application of the high power microwave generators, the ideal pulse voltage is the flattop rectangular pulse with fast rise time and fall time, and the plateau time of the main pulse voltage should be long enough, because the typical HPMG can operate at the highest efficiency in this pulse voltage [11]. Now the duration of the output voltage of the IEBA is increased by the development of the spiral BPFL [12-14]. However, for the Blumlein generator, the output voltage is usually a well-shaped square wave, but there is $[8,15,16]$ fluctuation on the flat part of the output voltage, which affects the quality of the electron beam. In the paper [16], the designed condition of spiral BPFL for the output ideal plateau voltage pulse at the matched load has been discussed. The effect of the fluctuation of the output voltage on other factors has not been investigated by others, so in this paper we will discuss the factors which affect the fluctuation of flat of the output voltage of IEBA, especially the transition section which is between the BPFL and load. In this paper, during the BPFL discharge into the load, the model of IEBA is presented and the factor which affects the flat of the output voltage of the IEBA is analyzed in theory; formulas for calculating the main pulse of IEBA are obtained. At last, experiments are performed on an IEBA based on BPFL with water dielectric. The experimental results accord with the theoretical results. The work in this paper is helpful to understand the fluctuation on the flat of the output voltage of IEBA based on BPFL, and the resulting equation for the output voltage pulse is useful to other researchers.

\section{THEORETICAL ANALYSIS}

\section{A. Model and theoretical calculation}

The construction of the IEBA based on the pulsed transformer and BPFL is illustrated in Fig. 1, which consists of the primary storage capacitor, field distortion switch, pulsed transformer, main switch, the water BPFL, transition section, the load, and resistive divider.

The BPFL consists of three coaxial cylindrical lines filled with deionized water as dielectric. The PFL formed by the inner cylinder and the middle cylinder are usually called inner line. If the PFL are formed by the middle 


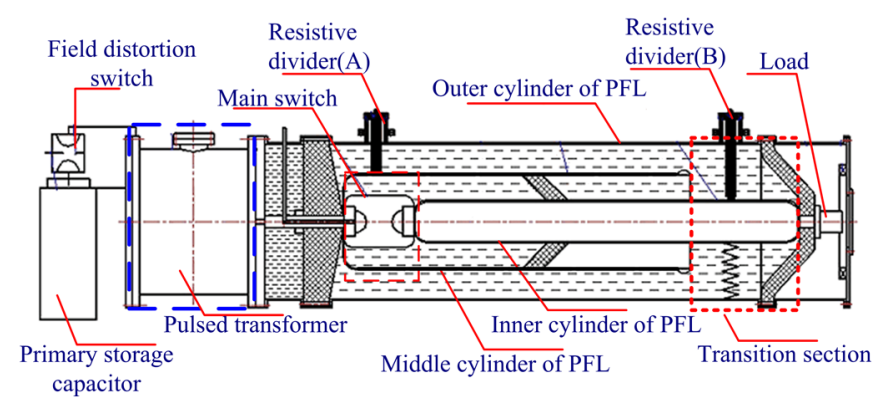

FIG. 1. (Color) Diagram of the IEBA based on the pulsed transformer and BPFL.

cylinder and the outer cylinder, they are called outer line. The transmission time and wave impedance of the inner line and outer line are $\tau_{1}, Z_{1}$ and $\tau_{1}, Z_{3}$, respectively. Also the transition section (Fig. 1) which is between the BPFL and the load is also a transmission line; it is formed by the inner cylinder and outer cylinder of the BPFL and the transmission time and wave impedance are $\tau_{2}, Z_{2}$. So during the BPFL discharge into the load, the equivalent circuit of the BPFL is shown in Fig. 2, where $S$ is the main switch, $C E$ is the inner line, $F D$ is the outer line, $E A$ and $B F$ are transition sections, and $Z_{L}$ is the load.

In order to get the output voltage of the IEBA, coordinate system $X, Y, Z$, and $W$ are built in Fig. 2. When the BPFL is charged to a voltage $U(U<0)$, once the main switch $S$ is closed, the BPFL cannot stay in equilibrium since the BPFL elementary capacitors adjacent to the load $Z_{L}$ start discharging. So the voltage at the inner line and outer line satisfied the telegraph equations:

$$
\begin{gathered}
-\frac{\partial u_{1}(X, t)}{\partial X}=L_{1} \frac{\partial i_{1}(X, t)}{\partial t} \quad-\frac{\partial i_{1}(X, t)}{\partial X}=C_{1} \frac{\partial u_{1}(X, t)}{\partial t} \\
-\frac{\partial u_{2}(Y, t)}{\partial Y}=L_{2} \frac{\partial i_{2}(Y, t)}{\partial t} \quad-\frac{\partial i_{2}(Y, t)}{\partial Y}=C_{2} \frac{\partial u_{2}(Y, t)}{\partial t} \\
-\frac{\partial u_{3}(Z, t)}{\partial Z}=L_{2} \frac{\partial i_{3}(Z, t)}{\partial t} \quad-\frac{\partial i_{3}(Z, t)}{\partial Z}=C_{2} \frac{\partial u_{3}(Z, t)}{\partial t} \\
-\frac{\partial u_{4}(W, t)}{\partial W}=L_{3} \frac{\partial i_{4}(W, t)}{\partial t} \quad-\frac{\partial i_{4}(W, t)}{\partial W}=C_{3} \frac{\partial u_{4}(W, t)}{\partial t},
\end{gathered}
$$

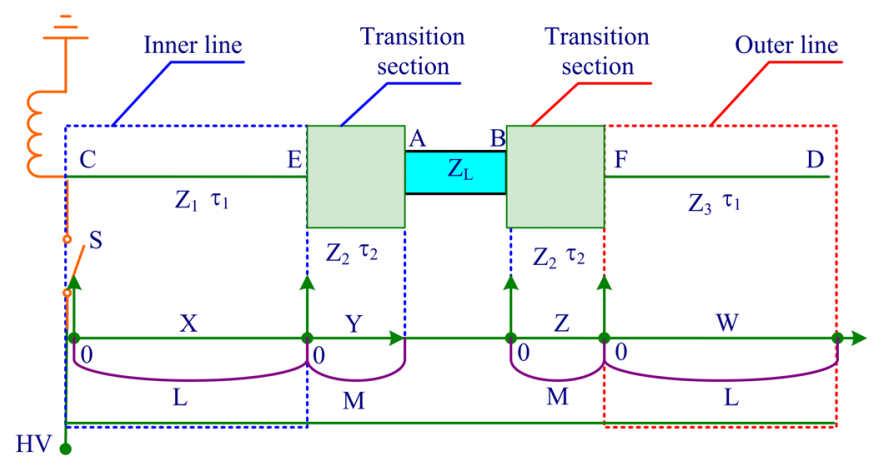

FIG. 2. (Color) Equivalent circuit of the BPFL discharge into the load.

where $u_{1}(X, t), i_{1}(X, t), u_{2}(Y, t), i_{2}(Y, t), u_{3}(Z, t), i_{3}(Z, t)$, $u_{4}(W, t)$, and $i_{4}(W, t)$ are voltage and current of the inner line, transition section and outer line, respectively. $L_{1}, L_{2}$, $L_{3}, C_{1}, C_{2}$, and $C_{3}$ are inductance and capacitance of per unit of the inner line, transition section, and outer line, respectively. For the characteristic of the BPFL in Fig. 2, the initial and boundary conditions of the BPFL are

point $\mathrm{C} \rightarrow u_{1}(0, t)=0$

point $\mathrm{E} \rightarrow i_{1}(L, t)=i_{2}(0, t)$

$$
u_{1}(L, t)=u_{2}(0, t)
$$

point $\mathrm{A} \rightarrow i_{2}(M, t)=i_{3}(0, t)$

$$
u_{2}(M, t)-u_{3}(0, t)=i_{3}(0, t) \times Z_{L}
$$

point $\mathrm{F} \rightarrow i_{3}(M, t)=i_{4}(0, t)$

$$
u_{3}(M, t)=u_{4}(0, t)
$$

point $\mathrm{D} \rightarrow i_{4}(L, t)=0$.

Then Laplace transform is used to solve Eq. (1). So the current and voltage is

$$
\begin{aligned}
& i_{1}(X, p)=A_{1} e^{-\left(p \tau_{1} / L\right) X}+B_{1} e^{\left(p \tau_{1} / L\right) X} \rightarrow u_{1}(X, p)=\frac{V_{B}}{p}+Z_{1}\left(A_{1} e^{-\left(p \tau_{1} / L\right) X}-B_{1} e^{\left(p \tau_{1} / L\right) X}\right) \\
& i_{2}(Y, p)=A_{2} e^{-\left(p \tau_{2} / M\right) Y}+B_{2} e^{\left(p \tau_{2} / M\right) Y} \rightarrow u_{2}(Y, p)=\frac{V_{B}}{p}+Z_{2}\left(A_{2} e^{-\left(p \tau_{2} / M\right) Y}-B_{2} e^{\left(p \tau_{2} / M\right) Y}\right) \\
& i_{3}(Z, p)=A_{3} e^{-\left(p \tau_{2} / M\right) Z}+B_{3} e^{\left(p \tau_{2} / M\right) Z} \rightarrow u_{3}(Z, p)=\frac{V_{B}}{p}+Z_{2}\left(A_{3} e^{-\left(p \tau_{2} / M\right) Z}-B_{3} e^{\left(p \tau_{2} / M\right) Z}\right) \\
& i_{4}(W, p)=A_{4} e^{-\left(p \tau_{1} / L\right) W}+B_{4} e^{\left(p \tau_{1} / L\right) W} \rightarrow u_{4}(W, p)=\frac{V_{B}}{p}+Z_{3}\left(A_{4} e^{-\left(p \tau_{1} / L\right) W}-B_{4} e^{\left(p \tau_{1} / L\right) W}\right),
\end{aligned}
$$

where $A_{1}, A_{2}, A_{3}, A_{4}, B_{1}, B_{2}, B_{3}$, and $B_{4}$ are undetermined coefficients, $p$ is the parameter in the Laplace transform. $\tau_{1}=L \sqrt{L_{1} C_{1}}=L \sqrt{L_{3} C_{3}}, \tau_{2}=M \sqrt{L_{2} C_{2}}$. Then substituting Eq. (3) into Eq. (2), and $x=e^{p \tau_{1}}, y=e^{p \tau_{2}}, A_{2}, B_{2}$ are 


$$
\begin{aligned}
& A_{2}=\frac{-2 V_{B}\left(\Phi x^{3} y^{4}+\Psi x y^{4}+\Gamma x^{3} y^{2}+\mathrm{K} x y^{2}\right)}{p\left(\alpha x^{4} y^{4}+\beta x^{2} y^{4}+\gamma x^{4} y^{2}+\varepsilon x^{4}+\xi y^{4}+\lambda x^{2}+\omega y^{2}+\eta\right)} \\
& B_{2}=\frac{2 V_{B}\left(\mathrm{~T} x^{3} y^{2}+\mathrm{N} x^{3}-\Gamma x y^{2}+\Lambda x\right)}{p\left(\alpha x^{4} y^{4}+\beta x^{2} y^{4}+\gamma x^{4} y^{2}+\varepsilon x^{4}+\xi y^{4}+\lambda x^{2}+\omega y^{2}+\eta\right)}
\end{aligned}
$$

and there are

$$
\begin{aligned}
& \alpha=Z_{L}\left(Z_{1}+Z_{2}\right)\left(Z_{2}+Z_{3}\right)+2\left[Z_{1} Z_{2}\left(Z_{2}+Z_{3}\right)+Z_{3}\left(Z_{2}^{2}+Z_{3}\right)\right] \quad \beta=-2 Z_{2}\left(Z_{L}+2 Z_{2}\right)\left(Z_{1}-Z_{3}\right) \\
& \gamma=2 Z_{L}\left(Z_{2}^{2}-Z_{1} Z_{3}\right) \quad \varepsilon=-Z_{L}\left(Z_{1}-Z_{2}\right)\left(Z_{2}-Z_{3}\right)+2\left[Z_{1} Z_{2}\left(Z_{2}-Z_{3}\right)+Z_{3}\left(Z_{2}^{2}-Z_{3}\right)\right] \\
& \xi=Z_{L}\left(Z_{1}-Z_{2}\right)\left(Z_{2}-Z_{3}\right)+2\left[Z_{1} Z_{2}\left(Z_{2}-Z_{3}\right)+Z_{3}\left(Z_{2}^{2}-Z_{3}\right)\right] \quad \lambda=2 Z_{2}\left(Z_{L}-2 Z_{2}\right)\left(Z_{1}-Z_{3}\right) \\
& \omega=-2 Z_{L}\left(Z_{2}^{2}-Z_{1} Z_{3}\right) \quad \eta=-Z_{L}\left(Z_{1}+Z_{2}\right)\left(Z_{2}+Z_{3}\right)+2\left[Z_{1} Z_{2}\left(Z_{2}+Z_{3}\right)+Z_{3}\left(Z_{2}^{2}+Z_{3}\right)\right] \\
& \Phi=\left(Z_{L}+2 Z_{2}\right)\left(Z_{2}+Z_{3}\right) \quad \Psi=-Z_{2}\left(Z_{L}-2\right)-2 Z_{2}^{2}+Z_{L} Z_{3} \quad \Gamma=Z_{L}\left(Z_{2}-Z_{3}\right) \quad \mathrm{K}=-Z_{L}\left(Z_{2}+Z_{3}\right) \\
& \mathrm{T}=Z_{L}\left(Z_{2}+Z_{3}\right) \quad \mathrm{N}=\left(Z_{L}-2 Z_{2}\right)\left(Z_{2}-Z_{3}\right) \quad \Lambda=-\left(Z_{L}-2 Z_{2}\right)\left(Z_{2}+Z_{3}\right)
\end{aligned}
$$

So the output voltage of IEBA is

$$
u_{Z_{L}}(p)=\frac{2 V_{B} Z_{L}}{p \alpha} \frac{\left[(\mathrm{T}-\Phi) x^{-1} y^{-1}+(\mathrm{N}-\Gamma) x^{-1} y^{-3}-(\Gamma+\Psi) x^{-3} y^{-1}+(\Lambda-\mathrm{K}) x^{-3} y^{-3}\right]}{1+\frac{\beta}{\alpha} x^{-2}+\frac{\gamma}{\alpha} y^{-2}+\frac{\varepsilon}{\alpha} y^{-4}+\frac{\xi}{\alpha} x^{4}+\frac{\lambda}{\alpha} x^{-2} y^{-4}+\frac{\omega}{\varepsilon} x^{-4} y^{-2}+\frac{\eta}{\alpha} x^{-4} y^{-4}} .
$$

For Eq. (8), we assume that

$$
\Theta=(\mathrm{T}-\Phi) x^{-1} y^{-1}+(\mathrm{N}-\Gamma) x^{-1} y^{-3}-(\Gamma+\Psi) x^{-3} y^{-1}+(\Lambda-\mathrm{K}) x^{-3} y^{-3}
$$

So Eq. (8) can be rewritten as

$$
u_{Z_{L}}(p)=\frac{2 V_{B} Z_{L} \Theta}{p \alpha} \sum_{n=0}^{\infty}\left[(-1)^{n} \sum_{i=0}^{n} C_{n}^{i}\left(\frac{\gamma}{\alpha} y^{-2}+\frac{\varepsilon}{\alpha} y^{-4}\right)^{i}\left(\frac{\beta}{\alpha} x^{-2}+\frac{\xi}{\alpha} x^{-4}+\frac{\lambda}{\alpha} x^{-2} y^{-4}+\frac{\omega}{\varepsilon} x^{-4} y^{-2}+\frac{\eta}{\alpha} x^{-4} y^{-4}\right)^{n-i}\right] .
$$

For the IEBA, the main pulse is the most important and we just pay attention to the flat of the main pulse of the output voltage of IEBA. So during the inverse Laplace transformation, just $x^{-1}$ is taken into account. Then Eq. (10) can be further simplified as

$$
u_{Z_{L}}^{1}(p)=\frac{2 V_{B} Z_{L}}{p \alpha}\left\{\sum_{n=0}^{\infty}(-1)^{n} \sum_{i}^{n} \frac{n !}{i !(n-i) !}\left(\frac{\gamma}{\alpha}\right)^{i}\left(\frac{\varepsilon}{\alpha}\right)^{n-i}\left[(\mathrm{~T}-\Phi) x^{-1} y^{-(4 n-2 i+1)}+(\mathrm{N}-\Gamma) x^{-1} y^{-(4 n-2 i+3)}\right]\right\} .
$$

Then substituting $x=e^{p \tau_{1}}, y=e^{p \tau_{2}}$ into Eq. (11),

$$
u_{Z_{L}}^{1}(p)=\frac{2 V_{B} Z_{L}}{p \alpha}\left\{\sum_{n=0}^{\infty}(-1)^{n} \sum_{i}^{n} \frac{n !}{i !(n-i) !}\left(\frac{\gamma}{\alpha}\right)^{i}\left(\frac{\varepsilon}{\alpha}\right)^{n-i}\left[(\mathrm{~T}-\Phi) e^{-p\left[\tau_{1}+(4 n-2 i+1) \tau_{2}\right]}+(\mathrm{N}-\Gamma) e^{-p\left[\tau_{1}+(4 n-2 i+3) \tau_{2}\right]}\right]\right\}
$$

Using the characteristic of the inverse Laplace transformation,

$$
L^{-1}\left(\frac{e^{-p n \tau}}{p}\right)=l(t-n \tau)
$$

where $L^{-1}$ stands for inverse Laplace transformation, $l(t)$ is the step function, which is defined as follows:

$$
l(t)= \begin{cases}1 & t \geq 0 \\ 0 & t<0\end{cases}
$$

So the flat of output voltage of the IEBA is

$$
\begin{aligned}
u_{Z_{L}}^{1}(t)= & \frac{2 V_{B} Z_{L}}{\alpha}\left\{\sum_{n=0}^{\infty}(-1)^{n} \sum_{i}^{n} \frac{n !}{i !(n-i) !}\left(\frac{\gamma}{\alpha}\right)^{i}\left(\frac{\varepsilon}{\alpha}\right)^{n-i}\right. \\
& \times\left[(\mathrm{T}-\Phi) l\left(t-\tau_{1}-(4 n-2 i+1) \tau_{2}\right)\right. \\
& \left.\left.+(\mathrm{N}-\Gamma) l\left(t-\tau_{1}-(4 n-2 i+3) \tau_{2}\right)\right]\right\}
\end{aligned}
$$

In fact, according to the characteristic of the wave propagation, $n$ is definite and determined by $\tau_{1}$ and $\tau_{2}$, $n_{\max }=\left[\tau_{1} / \tau_{2}\right]$ so 


$$
\begin{aligned}
u_{Z_{L}}^{1}(t)= & \frac{2 V_{B} Z_{L}}{\alpha}\left\{\sum_{n=0}^{\left[\tau_{1} / \tau_{2}\right]}(-1)^{n} \sum_{i}^{n} \frac{n !}{i !(n-i) !}\left(\frac{\gamma}{\alpha}\right)^{i}\left(\frac{\varepsilon}{\alpha}\right)^{n-i}\right. \\
& \times\left[(\mathrm{T}-\Phi) l\left(t-\tau_{1}-(4 n-2 i+1) \tau_{2}\right)\right. \\
& \left.\left.+(\mathrm{N}-\Gamma) l\left(t-\tau_{1}-(4 n-2 i+3) \tau_{2}\right)\right]\right\}
\end{aligned}
$$

Also, from Eq. (10), for $x^{-3} y^{-3}$, it is easy to get

$$
\begin{aligned}
u_{Z_{L}}^{2}(p)= & \frac{2 V_{B} Z_{L}}{\alpha}\left\{\mathrm{O}\left(\tau_{2}, \tau_{1}\right)+\left[(\Lambda-\mathrm{K})-(\mathrm{N}-\Gamma) \frac{\beta}{\alpha}\right.\right. \\
& \left.\left.+(\mathrm{B}+\Gamma) \frac{\gamma}{\alpha}+2(\mathrm{~T}-\Phi) \frac{\beta \gamma}{\beta^{2}}\right] x^{-3} y^{-3}\right\}
\end{aligned}
$$

Equation (17) determines the back edge of the output voltage, and $\mathrm{O}\left(\tau_{2}, \tau_{1}\right)$ stands for the remainder, if $t \leq$ $3 \tau_{1}+3 \tau_{2}, \mathrm{O}\left(\tau_{2}, \tau_{1}\right)=0$. So
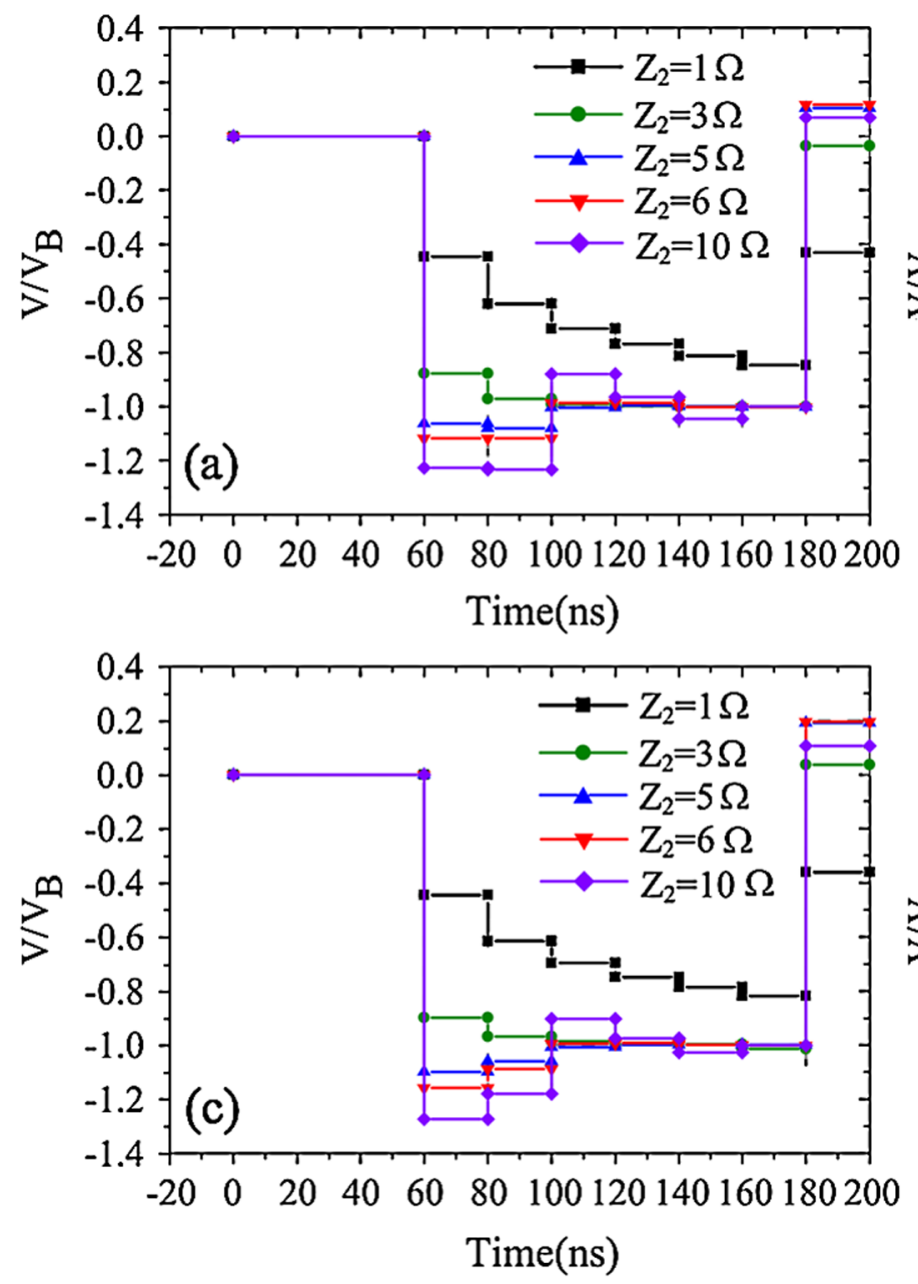

$$
\begin{aligned}
u_{Z_{L}}^{2}(t)= & \frac{2 V_{B} Z_{L}}{\alpha}\left\{\mathrm{O}\left(\tau_{2}, \tau_{1}\right)+\left[(\Lambda-\mathrm{K})-(\mathrm{N}-\Gamma) \frac{\beta}{\alpha}\right.\right. \\
& \left.\left.+(\mathrm{B}+\Gamma) \frac{\gamma}{\alpha}+2(\mathrm{~T}-\Phi) \frac{\beta \gamma}{\beta^{2}}\right] l\left(t-3 \tau_{1}-3 \tau_{2}\right)\right\}
\end{aligned}
$$

From Eqs. (16) and (18), the main pulse of the output voltage,

$$
\begin{aligned}
u_{Z_{L}}(t)= & \frac{2 V_{B} Z_{L}}{\alpha}\left\{\sum_{n=0}^{\left[\tau_{1} / \tau_{2}\right]}(-1)^{n} \sum_{i}^{n} \frac{n !}{i !(n-i) !}\left(\frac{\gamma}{\alpha}\right)^{i}\left(\frac{\varepsilon}{\alpha}\right)^{n-i}\right. \\
& \times\left[(\mathrm{T}-\Phi) l\left(t-\tau_{1}-(4 n-2 i+1) \tau_{2}\right)\right. \\
& \left.\left.+(\mathrm{N}-\Gamma) l\left(t-\tau_{1}-(4 n-2 i+3) \tau_{2}\right)\right]\right\} \\
& +\frac{2 V_{B} Z_{L}}{\alpha}\left\{\left[(\Lambda-\mathrm{K})-(\mathrm{N}-\Gamma) \frac{\beta}{\alpha}+(\mathrm{B}+\Gamma) \frac{\gamma}{\alpha}\right.\right. \\
& \left.\left.+2(\mathrm{~T}-\Phi) \frac{\beta \gamma}{\beta^{2}}\right] l\left(t-3 \tau_{1}-3 \tau_{2}\right)+\mathrm{O}\left(\tau_{2}, \tau_{1}\right)\right\} .
\end{aligned}
$$
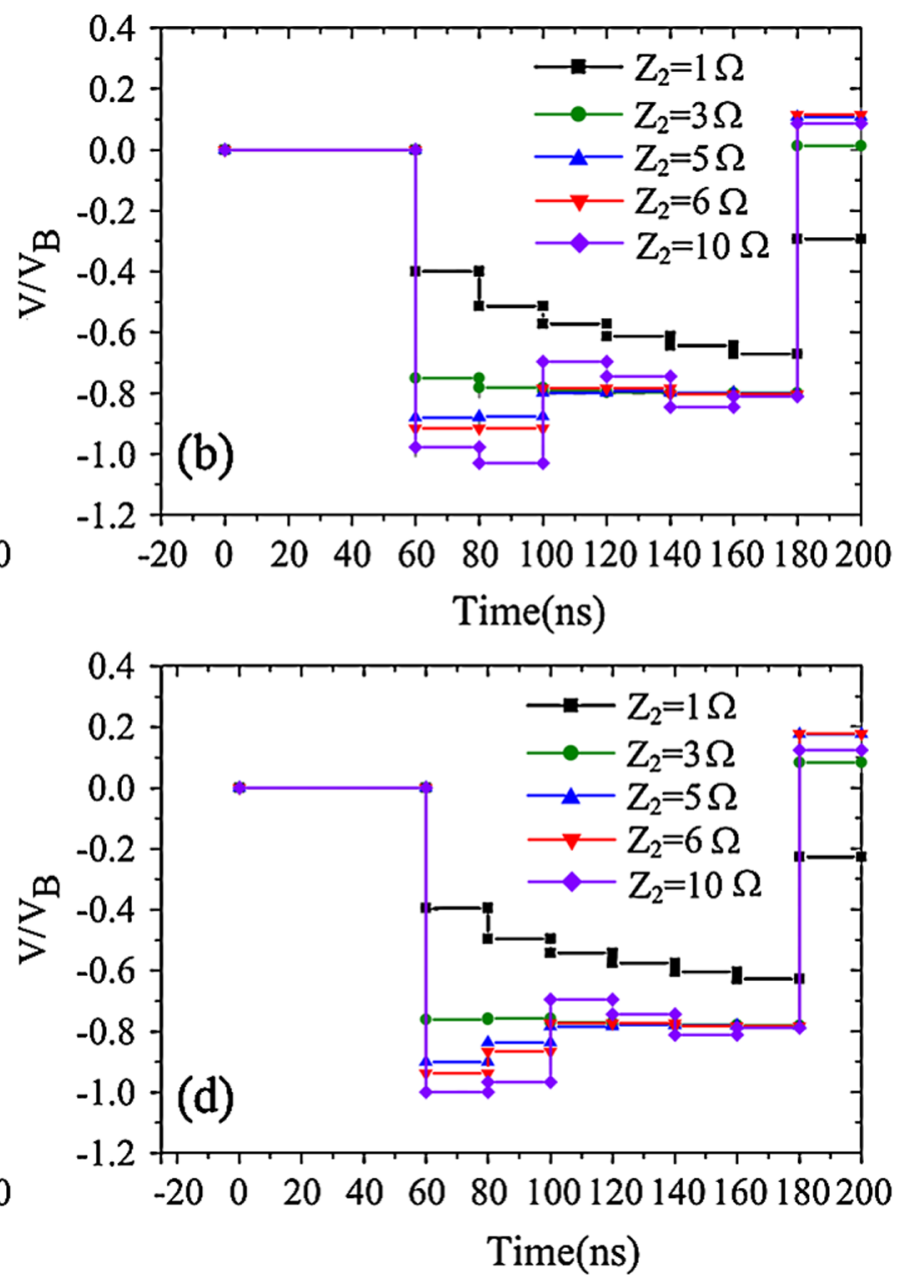

FIG. 3. (Color) The main pulse of the output voltage at different conditions, in all cases, $\tau_{1}=50 \mathrm{~ns}, \tau_{2}=10 \mathrm{~ns}$ : (a) $Z_{1}=Z_{3}=6 \Omega$, $Z_{L}=12 \Omega$; (b) $Z_{1}=Z_{3}=6 \Omega, Z_{L}=8 \Omega$; (c) $Z_{1}=6 \Omega, Z_{3}=8 \Omega, Z_{L}=14 \Omega$; (d) $Z_{1}=6 \Omega, Z_{3}=8 \Omega, Z_{L}=9 \Omega$. 
Equation (19) is the formula which is used to calculate the output voltage of the IEBA.

\section{B. Discussion with different conditions}

In order to show the voltage waveform directly, assuming that $\tau_{1}=50 \mathrm{~ns}, \tau_{2}=10 \mathrm{~ns}$, four conditions are discussed. (a) $Z_{1}=Z_{3}=6 \Omega, Z_{L}=12 \Omega$, which means that the load is matched with the BPFL completely. (b) $Z_{1}=Z_{3}=6 \Omega, Z_{L}=8 \Omega$, for this condition, the wave impedance of the inner line is equal to that of outer line, but the load is not mated with the BPFL. (c) $Z_{1}=6 \Omega, Z_{3}=8 \Omega, Z_{L}=14 \Omega$, although the load is matched with the BPFL, the wave impedance of the inner line is not equal to that of outer line. (d) $Z_{1}=6 \Omega$, $Z_{3}=8 \Omega, Z_{L}=9 \Omega$, for this condition, the load is not matched with the BPFL, also the wave impedance of the inner line is not equal to that of the outer line.

According to Eq. (19) and the assumption above, the main pulse of the output voltage at different conditions is
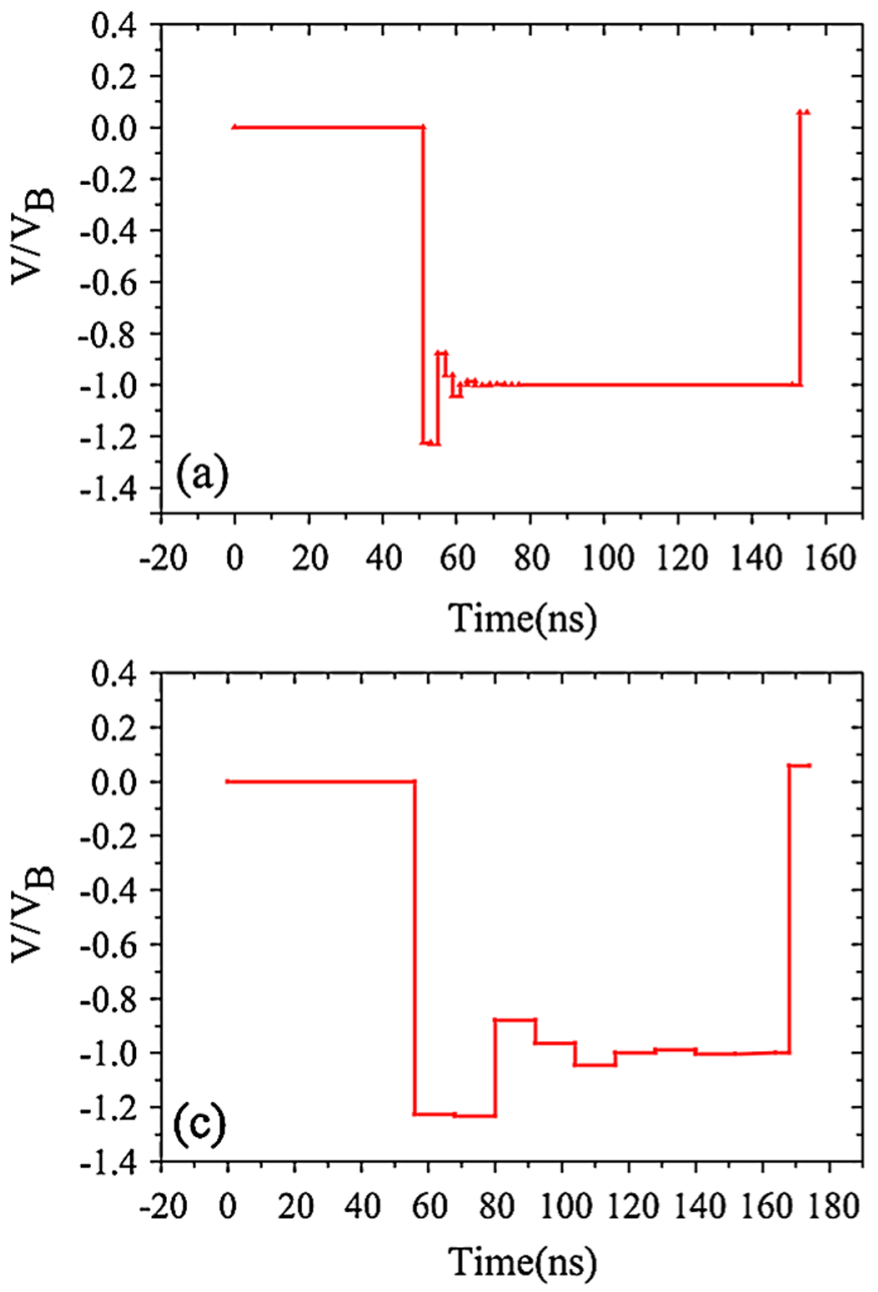

shown in Fig. 3. There is fluctuation on the flat part of the output voltage in Fig. 3, and when the wave impedance of the transition section is too small, there will be no flat at the output voltage. For example, when $Z_{2}=1 \Omega$, the output voltage is not a square wave at any conditions. Also, if the wave impedance of the transition section is too big, the fluctuation on the flat of the output voltage will be obvious, and the fluctuation increases with the incensement of the $Z_{2}$. Choosing the proper $Z_{2}$, there will be a minimal fluctuation. For example, In Figs. 3(b) and 3(d), if $Z_{2}$ is $3 \Omega$, the fluctuation of the main pulse is almost inexistent. So for the BPFL, a proper wave impedance of the transition section is very important for forming a well-shaped square wave.

One important thing should be noted: if the transmission time of the transition section is short, this kind of effect is not so obvious. For example, if $\tau_{1}=50 \mathrm{~ns}, Z_{2}=10 \Omega$ and $Z_{1}=Z_{3}=6 \Omega, Z_{L}=12 \Omega, \tau_{2}$ is $1,2,6$, and $10 \mathrm{~ns}$ separately. The main pulse of the output voltage of the BPFL is shown in Fig. 4.
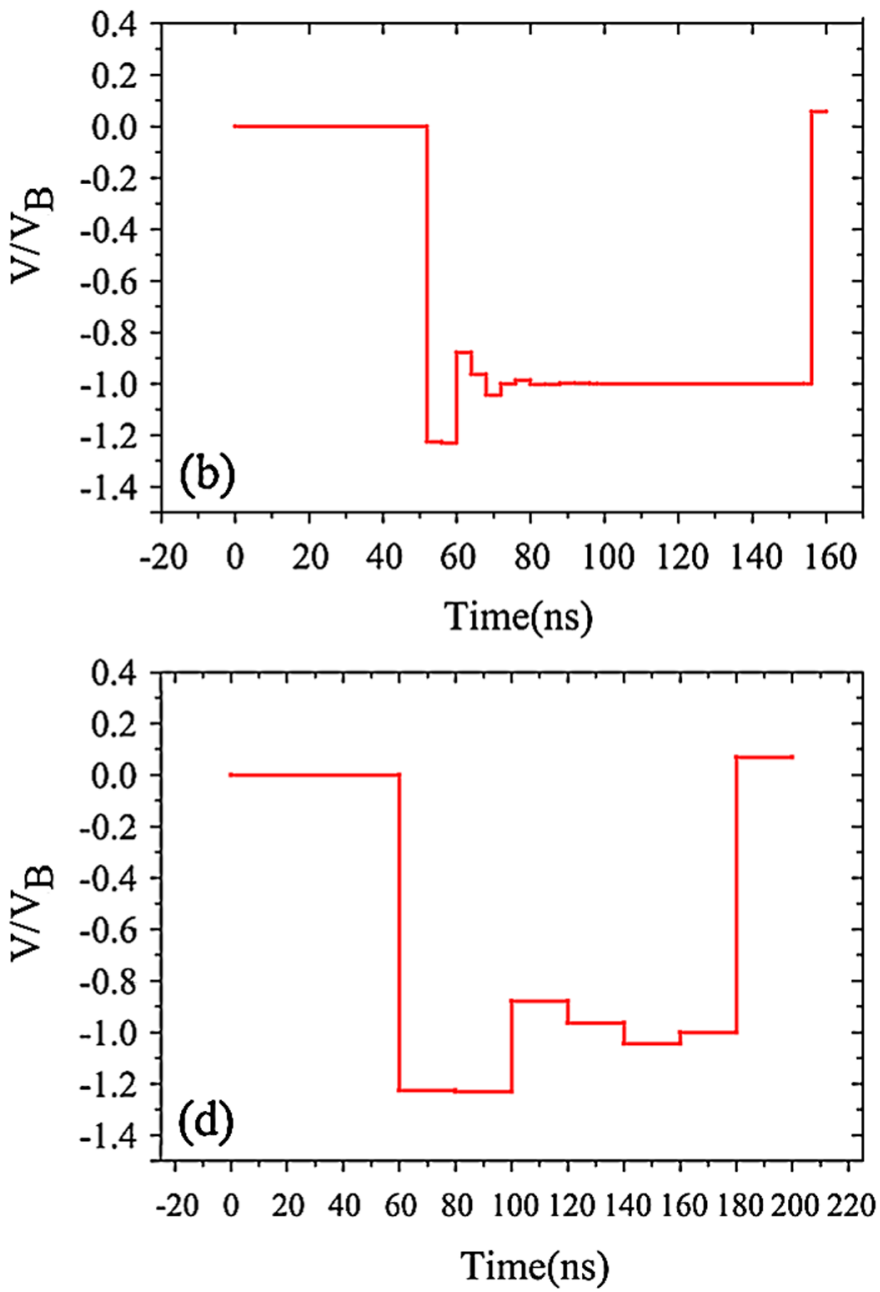

FIG. 4. (Color) The main pulse of the output voltage at different transmission time of the transition section, in all cases, $\tau_{1}=50 \mathrm{~ns}$, $Z_{1}=Z_{3}=6 \Omega, Z_{2}=10 \Omega, Z_{L}=12 \Omega$, the transmission time of the transition section is varied: (a) $\tau_{2}=1 \mathrm{~ns}$, (b) $\tau_{2}=2 \mathrm{ns,}$ (c) $\tau_{2}=6 \mathrm{~ns}$, (d) $\tau_{2}=10 \mathrm{~ns}$. 
In Fig. 4, when $\tau_{2}$ is 1 ns [Fig. 4(a)] and 2 ns [Fig. 4(b)], respectively, it shows that the output voltage is a wellshaped square wave, and the plateau time is about $90 \mathrm{~ns}$, $80 \mathrm{~ns}$. Therefore, the transition section has little effect on the output voltage. But if the transmission time increases from 2 to $6 \mathrm{~ns}$ [Fig. 4(c)], even $10 \mathrm{~ns}$ [Fig. 4(d)], the plateau time of the output voltage is only about 60 and $40 \mathrm{~ns}$, respectively; the duration of the fluctuation is much longer. So for the same BPFL, the transmission time of the transition section should be short, the shorter the better. Otherwise, the plateau time of the output voltage will be affected. Also, we know that the transmission time of the transition section is $\tau_{2}=M \frac{\sqrt{\varepsilon_{r}}}{c},\left(\varepsilon_{r}\right.$ is the relative permittivity of the dielectric of the BPFL, $c$ is the velocity of light), so the length of the transition section should be kept as short as possible.

Through that analyzed above, some basic conclusion can be obtained. (1) The wave impedance of the transition section should not be too small or too large, and a proper wave impedance of the transition section which is determined by the impedance of the BPFL is helpful to decrease the fluctuation of the flat of the output voltage and to form a well-shaped square wave. (2) For the same BPFL, in order to decrease the fluctuation of the flat of the output voltage, the transmission time of the transition section should be short, so the length of the transition section should be kept as short as possible.

\section{EXPERIMENTAL STUDIES AND DISCUSSION}

In order to identify the correctness of the theoretical analysis above, an experiment is performed using an IEBA [13] based on the strip spiral Blumlein PFL with water dielectric; the structure of the IEBA is shown in Fig. 1, and the IEBA is called IEBA-A. Through the theoretical calculation, the wave impedance of the inner line and outer line are $6.2 \Omega$ and $6.3 \Omega$, respectively, the transmission time $\tau_{1}$ is about $70 \mathrm{~ns}$. The wave impedance and transmission time of the transition section are $9 \Omega$ and $10 \mathrm{~ns}$, respectively. During the experiments, a high power load with inductance of $20 \mathrm{nH}$ and capacitance of $0.5 \mathrm{nF}$ is used, and the resistance of the load is adjustable. The output voltage of the IEBA is measured by 480:1 resistance voltage divider. Figure 5 shows the output voltage waveform when the load is $10 \Omega$.

In Fig. 5, it clearly shows that there is fluctuation on the flat of the output voltage, which may be caused by transition section. In order to verify this conclusion, substituting the parameters of the IEBA-A into the Eq. (19), the theoretical output voltage of the IEBA-A is shown in Fig. 6. Comparing with Fig. 5, the theoretical output voltage is in accordance with the experimental result well except for a little difference, because the stray inductance of the load is also one of factors which affects the fluctuation, but the transition section is the main reason which affects the

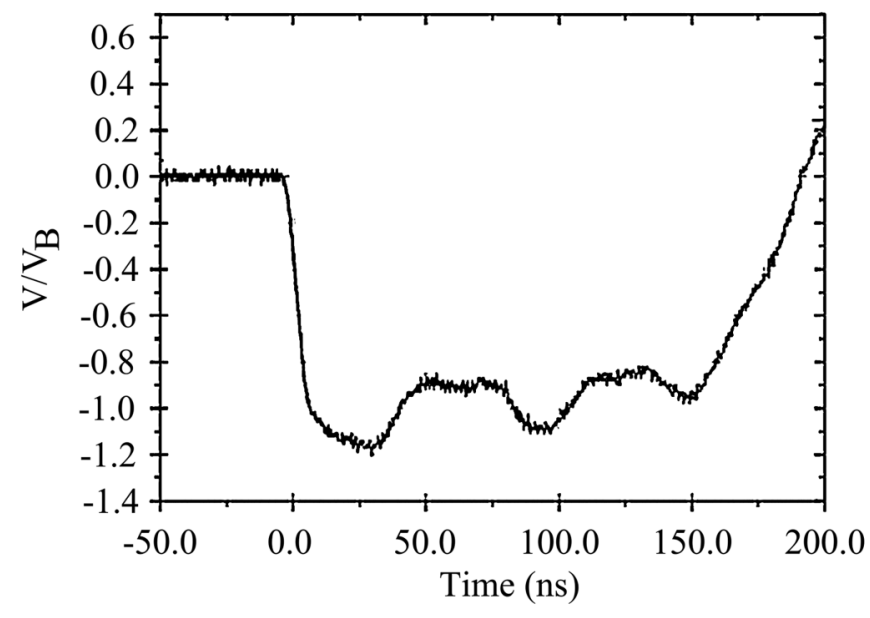

FIG. 5. Output voltage of the IEBA-A in the experiment, the parameters of the IEBA-A are $Z_{1}=6.2 \Omega, Z_{3}=6.2 \Omega, Z_{2}=$ $9 \Omega, Z_{L}=10 \Omega, \tau_{1}=70 \mathrm{~ns}, \tau_{2}=10 \mathrm{~ns}$.

fluctuation comparing between the experimental results and the theoretical results.

During the experiments, it is difficult to change the length of the transition section so, in order to verify the conclusion above, an experiment which is performed by Katsuki [6] is employed. In Ref. [6], an IEBA based on the BPFL is introduced: $\tau_{1}$ is about $35 \mathrm{~ns}$, the wave impedance of the inner line, outer line, and transition section are $5 \Omega$, $5.4 \Omega$, and $10.4 \Omega$, respectively, from the structure of the BPFL, $\tau_{2}$ is about $2 \mathrm{~ns}$, and the BPFL is charged by positive voltage of $120 \mathrm{kV}$. Now the Blumlein generator in Ref. [6] is called IEBA-B. Figure 7 shows the waveform of the output voltage of IEBA-B during the experiment when the load is $10 \Omega$. It is a well-shaped square waveform except for a sharp spike in front of the main pulse, and

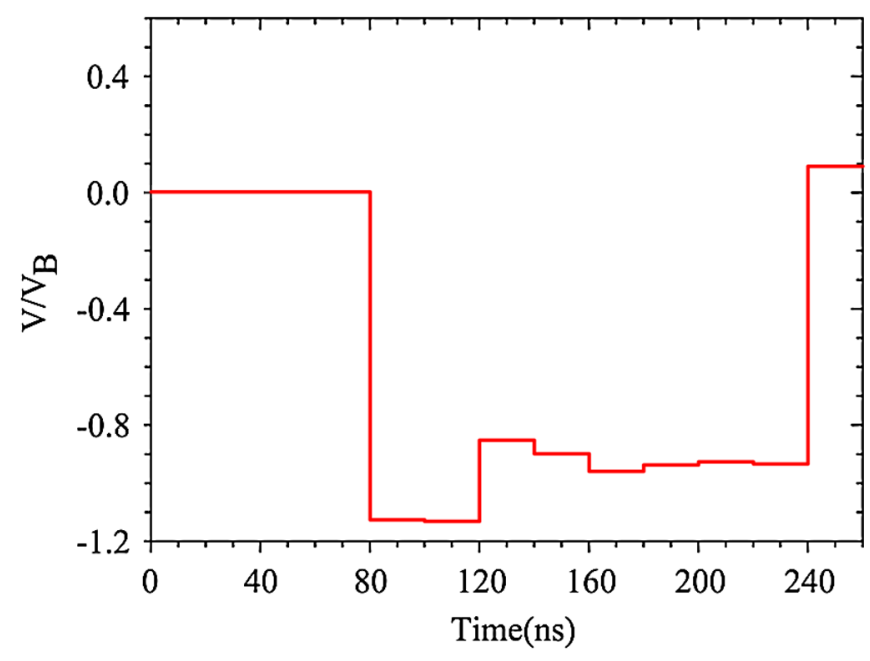

FIG. 6. (Color) Output voltage of the IEBA-A from the theoretical calculation when $Z_{1}=6.2 \Omega, Z_{3}=6.2 \Omega, Z_{2}=9 \Omega$, $Z_{L}=10 \Omega, \tau_{1}=70 \mathrm{~ns}, \tau_{2}=10 \mathrm{~ns}$. 


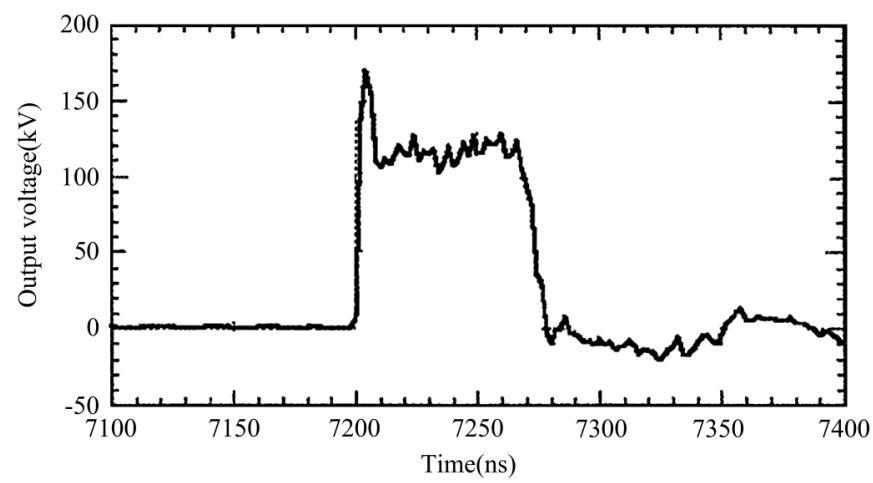

FIG. 7. Typical output voltage of the IEBA-B during the experiment in Ref. [6].

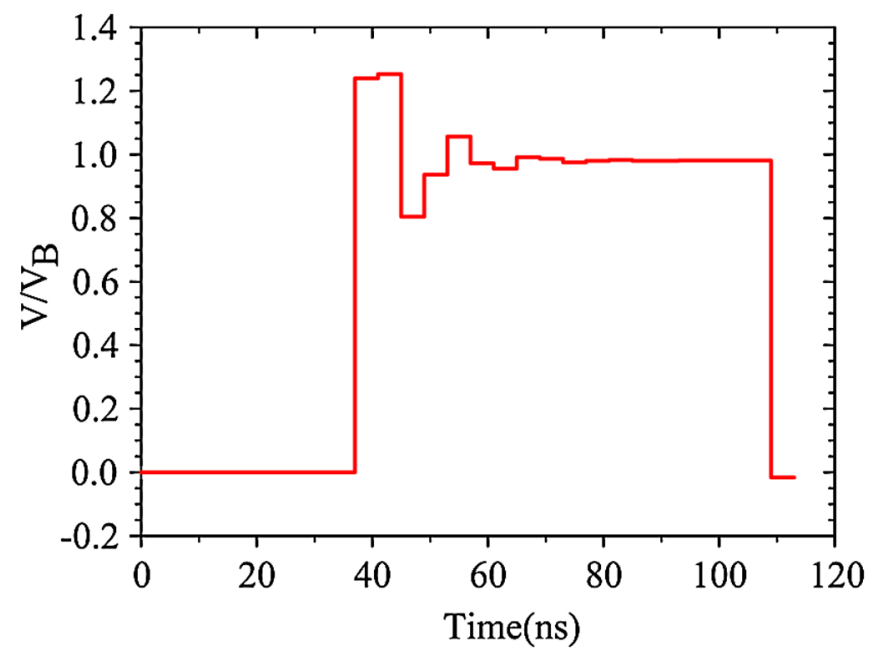

FIG. 8. (Color) Output voltage of the IEBA-B in Ref. [6] from the theoretical calculation.

Katsuki thinks that the sharp spike is caused by the stray inductance of the load.

Then substituting these parameters of IEBA-B into Eq. (19), the output voltage of IEBA-B in theory is shown in Fig. 8, and a sharp spike is formed in front of the main pulse. The voltage of the sharp spike is a little less than that of the experiment in Fig. 7, which may be caused by the stray inductance of the load. However, the flat of the output voltage is in accordance with the experiment result. So a conclusion can be drawn that the sharp spike is caused by the transition section as well as the stray inductance of the load.

Comparing the results between the IEBA-A and IEBA$B$, it clearly shows that the transition section between the BPFL and load has a great effect on the output voltage of the IEBA, and the experimental results correspond with the theoretical analysis well.

\section{CONCLUSION}

We have used the telegraph equations of BPFL to study the fluctuation of the output voltage of IEBA. Through the theoretical analysis, a formula which is used to calculate the output voltage of the IEBA based on the BPFL is obtained, and the factor which affects the fluctuation of the output voltage is analyzed. These results show that the output voltage of the IEBA is affected by the transition section of the BPFL seriously and some conclusions can be made as follows.

(1) Our calculating results in Fig. 3 demonstrate that the wave impedance of the transition section should not be too small or too large, and a proper wave impedance of the transition section which is determined by the impedance of the BPFL is helpful to decrease the fluctuation of the flat of the output voltage and to form a well-shaped square wave.

(2) For the same BPFL, in order to decrease the fluctuation of the flat of the output voltage, the transmission time of the transition section should be short enough, so the length of the transition section should be kept as short as possible. The shorter the transmission time of the transition section, the smaller the fluctuation, which is shown in Fig. 4.

At last, some experiments are used to verify the conclusions above, and the experiment results show reasonable agreement with the theoretical analysis. So, in order to decrease fluctuation of the output voltage of the IEBA based on the BPFL and to improve the electron-beam quality of such an accelerator, the transmission time of the transition section should be designed as short as possible.

\section{ACKNOWLEDGMENTS}

The authors gratefully acknowledge support from the National Natural Science Foundation of China under Grant No. 10675168. This work is also supported in part by Fund of innovation, Graduate school of NUDT, under Grant No. B090701.

[1] A.D. Blumlein, U.S. Patent No. 2,496,979 (7 February 1950).

[2] S. T Pai and Qi Zhang, Introduction to High Power Pulse Technology (World Scientific, Singapore, 1995).

[3] Sakugawa, T. Wang, D. Shinozaki, K. Namihira, T. Katsuki, S. Akiyama, and H. Graduate, Pulsed Power Conference, 2003. Digest of Technical Papers. 14th IEEE International (IEEE, New York, 2003), pp. 657-660.

[4] Yuanli Cheng, Yongpeng Zhao, Bohan Luan, Yan Li, Qiushi Zhu, and Qi Wang, 8th International Conference on Laser and Fiber-Optical Networks Modeling (IEEE, Kharkiv, Ukraine, 2006), pp. 62-65.

[5] S. Friedman, R. Limpaecher, and M. Sirchis, IEEE Conference Record of the Eighteenth Power Modulator Symposium (IEEE, Hilton Head, SC, 1988), pp. 360-366.

[6] S. Katsuki, D. Takano, T. Namihira, and H. Akiyama, Rev. Sci. Instrum. 72, 2759 (2001).

[7] J.L. Liu, C.L. Li, J.D. Zhang, S. Li, and X. X. Wang, Laser Part. Beams 24, 358 (2006). 
[8] J. Mondal, D. D. P. Kumar, A. Roy, S. Mitra, A. Sharma, S. K. Singh, G. V. Rao, K. C. Mittal, K. V. Nagesh, and D. P. Chakravarthy, J. Appl. Phys. 101, 034905 (2007).

[9] Steven H. Gold and Gregory S. Nusinovich, Rev. Sci. Instrum. 68, 3945 (1997).

[10] J. J. Coogan, F. Davanloo, and C. B. Collins, Rev. Sci. Instrum. 61, 1448 (1990).

[11] J. B. Robert and S. Edl, High Power Microwave Sources and Technologies, Institute of Electrical and Electronics Engineers, Inc. (Tsinghua University Press, Beijing, 2005) (in Chinese).

[12] S. D. Koroein, V. P. Gubanov, A. V. Gunin, I. V. Pegel, and A.S. Stepchenko, The 28th IEEE International Conference on Plasma Science, 2001, pp. 1229-1251.
[13] K. Yatsui, K. Shimiya, K. Masugata, M. Shigeta, and K. Shibata, Laser Part. Beams 23, 573 (2005).

[14] J. L. Liu, Y. Yin, B. Ge, T. W. Zhan, X. B. Cheng, J. H. Feng, T. Shu, J. D. Zhang, and X.X. Wang, Laser Part. Beams 25, 593 (2007).

[15] D. Durga Praveen Kumar, S. Mitra, K. Senthil, Archana Sharma, K. V. Nagesh, S. K. Singh, J. Mondal, Amitava Roy, and D. P. Chakravarthy, Rev. Sci. Instrum. 78, 115107 (2007).

[16] J. L. Liu, X. B. Cheng, B. L. Qian, B. Ge, J. D. Zhang, and X. X. Wang, Laser Part. Beams 27, 95 (2009). 